\title{
Equatorial wrinkles in the human lens capsule
}

\author{
H.J. Burd ${ }^{a,},{ }^{*}$, G. A. Montenegro ${ }^{b}$, L. Panilla Cortés ${ }^{b}$, R. I. Barraquer ${ }^{b, c}$, R. Michael ${ }^{b}$ \\ ${ }^{a}$ Department of Engineering Science, University of Oxford, Parks Road, Oxford OX1 3PJ, UK \\ ${ }^{b}$ Institut Universitari Barraquer, Universitat Autonoma de Barcelona, Barcelona, Spain \\ ${ }^{c}$ Centro de Oftalmología Barraquer, Universitat Internacional de Catalunya, Barcelona,Spain \\ * Corresponding author. Email: harvey.burd@eng.ox.ac.uk
}

\section{ABSTRACT}

Equatorial wrinkles, or crenations, have been previously observed around the equator in coronal images of the human ocular lens. However, wrinkles are typically not apparent when the lens is viewed from saggital directions. In the current paper, the existence and geometry of these wrinkles is shown to be consistent with a mechanical model of the isolated lens, in which the capsule is held in a state of residual tension by a spatially uniform internal pressure. The occurrence of equatorial wrinkles is therefore seen to be a mechanical consequence of the spheroidal shape of the lens capsule and an excess intralenticular pressure. New observations are made, on post mortem lenses, on the geometric arrangement of these equatorial wrinkles. These observations indicate a welldefined pattern in which wrinkles exists along meridional lines in the equatorial regions of the lens. A preliminary 'puncture test' is used to demonstrate that the residual stresses within the capsule in the equatorial region of the lens are broadly consistent with the proposed mechanical model of the lens capsule. It is suggested that the presence of equatorial wrinkles may have an influence on the mechanical performance of the capsule during the accommodation process.

\section{Introduction}

\subsection{Purpose}

Coronal images ${ }^{1}$ of the human lens that are available in the research and academic literature typically indicate the presence of equatorial undulations (e.g. Figs 1, 2 and 4 of Michael et al. (2008); Figs. 12.2 and 12.3 of Bron et al. 1997; Figs 9, 10, 13 and 18 of McCulloch (1954). These undulations are referred to by McCulloch (1954) as 'crenations'. Although the existence of these equatorial undulations has been documented previously, the mechanism that is responsible for their presence and the influence that they might have on the accommodation performance of the lens appears to have received little attention. In the current paper, it is demonstrated that these equatorial undulations are associated with systems of equatorial wrinkles aligned parallel to meridians ${ }^{2}$ on the lens surface.

\footnotetext{
${ }^{1}$ In this paper, the term 'coronal' is used to refer to images obtained by viewing the lens from a point on, or near to, the optical axis.

${ }^{2}$ It is assumed in this study that the surface of the ocular lens is a surface of revolution. The axis of revolution is referred to as the 'polar axis'. The term 'meridian' relates to any intersection of the lens surface with a plane containing the polar axis.
} 
The motivation for conducting a study of lens capsule wrinkles is as follows. Equatorial wrinkles are likely to reduce the mechanical resistance of the capsule to radial forces imposed on the lens, by the zonules, during the accommodation process. Previous mechanical models of the lens (e.g. the finite element models described in Burd et al. (2002); Hermans et al. (2006); Hermans et al. (2008); Stachs, et al. (2006); Weeber et al. (2007) are generally based on the assumption that the capsule is wrinklefree in the reference configuration adopted in the analysis (which is typically based on the fullyaccommodated state). Computational models of this sort may overestimate the radial forces that are applied in vivo to the lens when accommodation is relaxed, since any wrinkles that are present are likely to reduce the resistance of the lens capsule when radial stretching is applied via the zonules. To enable improved mechanical models of the accommodation process to be developed, data are needed on the geometric arrangement of any wrinkles that exist in the lens capsule. The mechanical influence of wrinkles can then be incorporated within the modelling process for the lens capsule (e.g. as discussed in Burd and Regueiro, 2015).

The presence of equatorial wrinkles may provide an explanation for the apparently anomalous equatorial displacements that are observed when human lenses are tested by spinning them on their polar axis. Fisher (1971), for example, noted that the capsule had an insignificant influence on the equatorial movements developed in the spinning lens (for the single de-capsulated lens that was tested at a rotational speed of $1000 \mathrm{rpm}$ ). This observation is inconsistent with a finite element analysis (Burd et al. 2006) in which the capsule is modelled as a wrinkle-free membrane. More recent spinning lens data (Burd et al. 2011; Wilde et al. 2012) indicate that the lens capsule does have a measurable constraining influence on the equatorial movements, but (unpublished) finite element calculations conducted by the first author suggest that this observed constraining effect is less significant than would be expected on the basis of a mechanical analysis in which the capsule is assumed to be taut (i.e. wrinkle-free) in the equatorial region.

The current investigation is concerned solely with equatorial wrinkles in isolated lenses. It is not intended to provide a complete study of lens capsule wrinkling. Instead, the purpose of the current paper is to consider a variety of evidence (from the existing literature, mechanical modelling and new observations) to explore the existence, cause and likely geometric arrangement of equatorial wrinkles in the human lens. Detailed studies of lens wrinkling, including the development of wrinkling with age and the influence that wrinkles might have on the accommodation performance of the lens, will form the basis of future work. 


\subsection{Evidence from the literature on lens equatorial wrinkles}

The coronal lens images, obtained from intact specimens (i.e. in which the lens is attached to the ciliary body via the zonules), in Fig 12.3 of Bron et al. (1997) and Fig. 2 of McCulloch (1954) provide particularly clear views of the equatorial undulations that are the subject of the current paper. Equatorial undulations are also evident in published images of isolated lenses, e.g. Fig. 2 of Michael et al. (2008) and the 63-year isolated lens shown in Fig. 2 (right) of Rosen et al. (2006) (reproduced in the current paper as Fig. 1 right); these image provides evidence that equatorial undulations can exist in the lens capsule in the absence of radial forces applied by the zonule.

In contrast, sagittal images ${ }^{3}$ of the lens typically appear to show an outline that is smooth around the entire lens perimeter, including in the equatorial region. Examples of images of this sort are given in Fig 12.1 of Bron et al. (1997) (for an intact specimen) and Fig. 2a of Rosen et al. (2006) (reproduced in this paper as Fig. 1 left) and Fig. 1 of Urs et al. ( 2009) for isolated lenses.

These images suggest that systems of wrinkles exist in the lens capsule in the equatorial region, such that wrinkles lie along meridians on the lens surface. These wrinkles appear as equatorial undulations in coronal lens images but, since they are oriented in meridional directions, they are not visible in sagittal views.

\section{Materials and Methods}

Four distinct studies have been conducted to investigate the geometric patterns of equatorial wrinkles in the human lens and to suggest a mechanism that is responsible for their presence. Initially, a mechanical model of the lens capsule - termed 'inflated membrane model'- is described. This model employs conventional methods from structural mechanics; it is used to explore a possible causal link between excess intralenticular pressure and the existence of equatorial wrinkles in the lens capsule.

Three separate experimental studies are then described. In the first study, a set of 185 coronal lens images - obtained from previous unrelated research projects - was subjected to a systematic visual assessment to classify the extent of equatorial wrinkling. Each lens in the set was classified as having a particular 'wrinkle grade'. Secondly, 4 human post mortem lenses L1-L4 - collected specifically for the purpose of the current research - were subjected to detailed quantitative assessments of equatorial wrinkle geometry. Each lens was mounted in a 'lens rotation box' and images of the lens edge were collected at different angular orientations of the lens. The geometric arrangement of the

\footnotetext{
${ }^{3}$ In the current paper, the term 'sagittal' is used to refer to images that are obtained by viewing the lens in a direction that is orthogonal to the polar axis. The outline of the lens when viewed in this way corresponds (approximately, depending on the imaging system being used) to a meridian.
} 
wrinkles was then determined by automatic analysis of the lens edge images. Thirdly, a preliminary 'puncture test' was conducted on a separate set of 9 presbyopic post mortem lenses, P1-P9. The test protocol involved making incisions in the capsule in the equatorial region of the lens; the observed capsule rupture patterns provide qualitative information on the nature of the residual stresses in the capsule. These observations are used to assess the assumptions inherent in the inflated membrane model.

The lenses tested in the lens rotation box (lenses L1-L4) and in the puncture test (lenses P1-P9) are specified in Table 1. Donor globes were stored in a refrigerator post mortem. To extract each lens, the cornea was initially removed from the globe with a trephine (for transplantation purposes.) The zonules were then cut with Westcott scissors and the lens was carefully removed with curved blunt forceps. Testing of each lens was conducted immediately after it had been extracted. Lenses for the set L1-L4 were selected from lenses with wrinkle grades of 2 or 3 . No other exclusion criteria (e.g. relating to the presence of cataract) were employed.

\section{Inflated membrane model}

It is hypothesised that the smooth profile of the sagittal outline of the isolated lens is maintained by an excess intralenticular pressure. The capsule is therefore held in a state of tension, in much the same way as an inflated balloon.

There is evidence in the literature to support the hypothesis that significant residual tensile stresses exist in the capsule of the isolated lens. Pedrigi et al. (2007), for example, suggest that the mechanical strain in the in situ capsule of an isolated human lens in the central anterior region is of the order of $3 \%$. These observed strains imply the existence of in-plane residual stresses which, in turn, suggest the presence of an excess intralenticular pressure. Furthermore, data in Krag and Andreassen (1996) indicate lens capsule residual strains of between $2.8 \%$ and $4 \%$ (although since these data were obtained from isolated porcine lenses, they may not be directly relevant to the human lens).

In mechanical terms, the lens consists of two distinct components; the capsule and the lens substance. The Young's modulus of the lens substance, particularly for young lenses, is known to be several orders of magnitude lower than the Young's modulus of the capsule. For a lens of age 20 years, for example, the Young's modulus of the cortex is estimated to be $0.386 \mathrm{kPa}$ (on the basis of Model D of Wilde et al. (2012) and an assumed Poisson's ratio of 0.5). The Young's modulus of the capsule, however, is some three orders of magnitude greater than this; it is estimated to be approximately $1000 \mathrm{kPa}$ (on the basis of uniaxial tests described by Krag and Andreassen (2003) or $6000 \mathrm{kPa}$ (on the basis of biaxial tests reported by Fisher 1969). These data support an approximate 
model of the isolated lens (referred to in this paper as the inflated membrane model) consisting of a structural membrane $e^{4}$ (the capsule) inflated by a spatially uniform excess intralenticular pressure, $p$. The key modelling assumption - that the excess intralenticular pressure acting on the capsule is uniform - is thought to be justified on the basis that the stiffness of the lens substance is small compared with the stiffness of the capsule (particularly for the young lens).

On the basis of the inflated membrane model, and assuming that the lens capsule is rotationally symmetric about the polar axis, it is straightforward to determine relationships between the principal stress resultants ${ }^{5}$ that exist in the capsule, the local radii of curvature and the magnitude of the excess intralenticular pressure. These relationships are given in Equations (1) and (2), in terms of the principal stress resultants $T_{\theta}$ acting in the meridional direction and $T_{\phi}$ acting in the circumferential direction. These stress resultants are shown diagrammatically in Fig 2.

The stress resultants can be expressed (e.g. Irvine 1981) as:

$T_{\theta}=\frac{1}{2} p r_{2}$

$T_{\phi}=\frac{1}{2} p r_{2}\left(2-\frac{r_{2}}{r_{1}}\right)$

where the local radius of curvature of the lens meridian is $r_{1}$, and $r_{2}$ is the distance to the polar axis along the normal to the capsule. Note the important result that the stress resultants are independent of the local capsule thickness. Equation (1) indicates that $T_{\theta}$ is positive (i.e. the meridional stress resultant is tensile) provided that the lens is convex $\left(r_{2}>0\right)$ and the excess intralenticular pressure, $p$, is positive. Both of these conditions are expected to hold in the normal human isolated lens. The existence of tensile meridional stress resultants is consistent with the occurrence (e.g. Fig. 1 left) of a smooth sagittal lens outline. Equation (2), however, shows that $T_{\phi}$ is tensile only if,

$\frac{r_{2}}{r_{1}}<2$

For regions of the lens capsule where the inequality (3) is not satisfied, the circumferential stress resultant, $T_{\phi}$, cannot be tensile. Equation (2) indicates that in these regions the circumferential stress resultant is compressive. Since the lens capsule is thin, it is unable to support significant compressive stresses without buckling ${ }^{6}$. Regions for which inequality (3) is not satisfied would

\footnotetext{
${ }^{4}$ The term 'membrane' is used in the current paper to describe a thin, structural element.

${ }^{5}$ The term 'stress resultant' is used in this paper to describe the product of the in-plane stress and the local capsule thickness.

${ }^{6}$ Buckling is a form of elastic instability that occurs in thin sheets or slender structural elements under the action of compressive forces.
} 
therefore be expected to exhibit a buckling instability. This causes wrinkles to develop that are aligned orthogonal to the direction of $T_{\phi}$ (i.e. aligned along the meridian).

For typical lens geometries, e.g. based on the sagittal profiles given in Urs et al. (2010), it is found that inequality (3) is satisfied in the central regions of the capsule (i.e. near the polar axis). Conversely, however, it is not satisfied near the equator. These observations suggest the capsule wrinkling model shown in Fig. 3. In zone I, inequality (3) is satisfied and the capsule is under a state of biaxial tension. In zone II, however, inequality (3) is not satisfied; tensile stresses act in the meridional direction, the capsule is buckled in the circumferential direction and $T_{\phi}$ is assumed to be zero. The location of the edges of the wrinkled zone (i.e. the interfaces between zones I and II) is determined by the condition $\frac{r_{2}}{r_{1}}=2$. In the capsule wrinkling model shown in Fig. 3, these edges are defined by the elevation angle, $\beta$, measured from the equatorial plane relative to point $\mathrm{C}$ (the intersection of the polar axis and the equatorial plane of the lens). The magnitude of the elevation angle at the interface of zones I and II is referred to in the current paper as 'wrinkle angle'; $\beta_{a}$ for the anterior surface and $\beta_{p}$ for the posterior surface.

Equations (1) and (2) only strictly apply in zone I where the outline is smooth and rotationally symmetric. Within zone II, the membrane is assumed to be buckled and these equations do not strictly apply. Although the inflated membrane model cannot describe the detailed mechanics of the wrinkles in zone II, it can be used to estimate the location of the interface between zones I and II.

To demonstrate the use of the model to estimate the wrinkle angles $\beta_{a}$ and $\beta_{p}$, an analysis has been conducted for the fully-accommodated (8D) in vivo 29-year lens meridional outline specified by Hermans et al. (2006); it is assumed that this outline is applicable to an isolated lens. A numerical analysis on this lens outline indicates that, on the basis of the inequality in Equation (3), $\beta_{a}=8^{\mathbf{0}}$ and $\beta_{p}=10^{\circ}$. Separate analyses on the Fourier series lens outlines for isolated human lenses given in Urs et al. (2010) indicate that, for lenses aged between 20 and 60 years, $\beta_{a}$ varies between $22^{\circ}$ and $25^{\circ}$ and $\beta_{p}$ varies between $15^{\circ}$ and $16^{\circ}$.

It is interesting to note that Irvine (1981) demonstrates that an inflated spheroidal membrane will inevitably exhibit equatorial wrinkles for an aspect ratio (equatorial diameter/polar thickness) greater than $\sqrt{2}$ (i.e. about 1.4). Furthermore, Irvine (1981) shows that the maximum aspect ratio that can be achieved (by optimising the shape of the meridional outline) for a wrinkle-free inflated rotationally symmetric inflated membrane is about 1.70 . According to Augusteyn (2008), normal isolated human lenses have an aspect ratio of between 2.2 and 2.3. On this basis, the inflated membrane model suggests that equatorial wrinkles would be expected to occur in all normal, isolated, human lenses, provided that an approximately uniform intralenticular pressure exists. 


\section{Initial observations on lens equatorial wrinkles in isolated human lenses}

In an initial study conducted on dark-field coronal images of a set of 185 isolated human lenses, equatorial wrinkles apparent in the images, determined 'by eye', were counted; no attempt was made to automate the identification of the wrinkles. The lenses were classified into four separate grading categories, as shown in Fig. 4, according to the number of wrinkles observed around the equator. The observed wrinkle gradings are shown in Fig. 5 (left) grouped according to age. The same data are shown in Figs 5 (centre) and 5 (right) grouped according to the LOCS III grading (Chylack et al. 1993) of cortical cataract and nuclear cataract respectively. These data suggest a general tendency for the number of wrinkles in the lens to decrease with increasing age. There does not appear to be a significant correlation between wrinkle grade and the presence of cortical or nuclear cataract. It is noteworthy that none of the lenses in the study were observed to be free of equatorial wrinkles.

\section{Detailed observations on equatorial wrinkles}

\subsection{Lens rotation box}

A specially designed lens rotation box (Fig. 6) was employed to rotate isolated lenses by angle $\psi_{r}$ about a horizontal axis. The lens rotation box consists of a rectangular perspex box with an open top. A horizontal shaft is connected to a cradle that holds the lens. A graded knob is used to rotate the shaft, manually, in angular increments of $3.6^{\circ}$. Lens images, obtained from a camera mounted directly above the box, for different rotation angles, $\psi_{r}$, were used to determine the way in which the equatorial wrinkles extend towards the lens poles. All observations were made with the lens immersed in BSS (balanced salt solution). Four lenses (L1-L4, see Table 1) were inspected using this system.

To prepare the sample, excess water was removed from the lens with a micro sponge. A handmade metallic ring ( $4.5 \mathrm{~mm}$ outer diameter, made from $1 \mathrm{~mm}$ diameter wire) was then glued, in air, with Histoacry $l^{\circledR}$ to the posterior surface of the lens. Thirty seconds after gluing the ring to the lens, the lens was immersed in BSS and attached, magnetically, to a magnetic strip fixed to the cradle

\subsection{Coordinate systems}

Analysis of the images is based on the set of right-handed coordinate systems shown in Fig. 7. Conventions used for polar coordinates are shown in Fig. 8.

\subsection{Imaging procedures}

Initially, a coronal image (corresponding to $\psi_{r}=0$ ) is obtained (e.g. as indicated in Fig 9a). The lens is then rotated clockwise in $3.6^{\circ}$ degree increments (in a negative sense, based on the conventional 
'right-hand rule') and lens profile images are collected at each rotational position (Fig 9b shows an example image for $\left.\psi_{r}=-46.8^{\circ}\right)$. The lens is rotated to $\psi_{r}=-90^{\circ}$ to image the sagittal profile; this image is used to determine whether the equatorial plane is tilted relative to the shaft. If tilt is observed, then the tilt angle $\alpha$ is estimated from the sagittal image and incorporated in the subsequent data processing procedures (see the Appendix). A typical sagittal profile is shown in Fig. 9c.

Next, the cradle is returned to the $\psi_{r}=0$ position. It is then rotated in $3.6^{\circ}$ degree increments in a positive sense (i.e. anticlockwise) and lens profile images are collected at each rotational position. This allows images of the posterior surface of the lens to be obtained.

These images are subsequently processed to track the anterior wrinkles for $\phi^{\prime \prime}=90^{\circ} \pm 50^{\circ}$ (i.e in the upper part of the image as indicated in Fig 9a) where $\phi^{\prime \prime}$ is azimuthal angle in the shaft coordinate system as the lens is rotated. Images for $\psi_{r}<0$ provide data on the wrinkles on the anterior surface of the lens and images for $\psi_{r}>0$ provide data on the posterior surface.

\subsection{Image and data processing}

A systematic approach was developed to identify the presence of wrinkles in the images. Initially, the images were converted to black and white and then analysed with bespoke software (developed by Aufried T.M. Lenferink, TWENSPEC-optical designs \& services, Stroweg 14, 7552BG Hengelo, Netherlands) to detect the lens outline. The lens outline was then filtered (using FFT) to remove low and high frequency components. The presence of wrinkles was determined automatically by searching for peaks in the data (using routines available in Matlab R2015b) with a prominence of 1.75 pixels or more (where the term 'prominence' refers to the minimum radial distance between a peak and one of its neighbouring troughs).

The wrinkle locations were further processed as follows. A 3D rotationally-symmetric model of the lens was assumed, for which the meridional outline is obtained from the age-dependent Fourier model in Urs et al. (2010). For each wrinkle location that is detected using the procedure described above, the spherical coordinates $(\beta, \phi)$ (in the lens coordinate system, $X)$ are determined using a numerical method (described in the Appendix) accounting for lens tilt (when present).

\subsection{Results}

Data that are obtained from this process, for lens L2, are plotted in Fig. 10. Fig. 10a indicates the filtered lens edge profiles obtained for positive (i.e anticlockwise) values of the rotation angle $\psi_{r}$. The data in Fig 10a are therefore limited to the anterior surface of the lens. Also shown, indicated 
with filled circles, are the peaks in the data (assumed to represent the presence of wrinkles) detected automatically using the procedure in Section 5.4.

The data have been further processed, on the basis of the lens outlines in Urs et al. (2010) to provide a representation, shown in Fig 10b, in which the relationship between azumithal angle and elevation angle for each detected wrinkle point is plotted. Fig $10 \mathrm{~b}$ indicates the presence of linear wrinkles extending towards the lens poles along the meridians (i.e. lines of constant azimuthal angle). The anterior wrinkles typically extend to values of elevation angle $\beta$ of about $5^{\circ}$ (although one of the wrinkles extends to $\beta=12^{0}$ ). The wrinkles appear shorter on the posterior surface. On the lens equator $(\beta=0)$ the 'wavelength', corresponding to the angular separation of the wrinkle peaks, is seen to be reasonably uniform with values of between $6^{\circ}$ and $9^{\circ}$. The wrinkles are seen to gradually disappear with increasing elevation angle; this same tendency was observed in the images obtained from all of the observations.

For each of the four lenses in the data set, a set of metrics was determined as follows:

(a) The number of wrinkle peaks detected in the coronal image, $\psi_{r}=0\left(\right.$ for $\phi=90^{\circ} \pm 50^{\circ}$ ) is recorded. For each peak the 'wavelength' is determined as the angular separation between the two adjacent peaks.

(b) The wrinkle angles (corresponding to the maximum elevation angle of the wrinkles; $\beta_{a}$ for the anterior surface and $\beta_{p}$ for the posterior surface) are determined as the largest value of elevation angle, $\beta$, for which a peak is detected in the data.

Metrics determined in this way are given in Table 2 . These data indicate a consistent pattern. Winkles exist at the lens equator with wavelengths of the order of $10^{\circ}$ (corresponding to a distance of about $0.8 \mathrm{~mm}$ around the lens circumference). The wrinkles extend towards the anterior and posterior poles along the lens meridians. The wrinkles gradually dissipate along the meridians, disappearing completely at an anterior wrinkle angle, $\beta_{a}$, of between $5^{0}$ and $12^{0}$ and at a posterior wrinkle angle, $\beta_{p}$, of between $3^{0}$ and $8^{0}$. For all of the lenses L1-L4, the anterior wrinkle angle is greater than the posterior wrinkle angle.

The wrinkle patterns observed in these tests are broadly consistent with the inflated membrane model as outlined in Section 3, although the general magnitude of the wrinkle angles are slightly less than those that are predicted by the Hermans et al. (2006) and the Urs et al. (2010) lens outlines. Consistent with predictions made using the Urs et al. (2010) outline (but not with the Hermans et al. (2006) outline), the posterior wrinkle angle is consistently observed to be less than the anterior wrinkle angle. 
As an additional experiment, a coronal image of lens $L 3$ was obtained after it had been de-capsulated (Fig. 11a) and compared with an image of the intact lens (Fig 11b). These images indicate that the removal of the capsule does not have a significant influence on the appearance of the wrinkles. It appears that, in this case, the lens substance has become moulded to the wrinkled shape of the capsule and that these moulded wrinkles persist after the capsule is removed.

\section{Puncture test}

The inflated membrane model described in Section 3 suggests that, in zone II, significant tensile stresses act in the capsule in the meridional direction, but any stresses in the circumferential direction are small (and compressive). The plausibility of a stress system of this sort acting in the equatorial region of the capsule has been explored by making incisions in the equatorial region of a set of post mortem lenses to observe whether the observed rupture modes are consistent with the stress conditions implied by the model. This procedure is referred to as a 'puncture test'.

Puncture tests were conducted on a set of 9 lenses (P1-P9, see Table 1). To conduct the test, each lens was by supported on a small amount of 'playdough' within a plastic dish. Incisions were then made on the lens equator in two orthogonal directions, using a V-shaped symmetrical lancet (V-Lance Knife, Alcon). Incisions were made either with the blade parallel to the equator or with the blade perpendicular to the equator (Fig. 12). Initially, two incisions were attempted; one on each side of the lens. It was discovered, however, that after the first incision had been made, capsule tension was lost and it was not possible to make an incision on the opposite side of the lens.

Initial experiments confirmed that an appropriate staining protocol would be required to visualise the ruptured edges of the capsule. After some experimentation it was found that the use of methylene blue and local red ink provided acceptable results; the stains were applied locally to the lens, immediately before the test, via droplets from a syringe. It proved impossible, however, to obtain clear photographic images of the edges of the capsule after it had been ruptured. Instead, hand drawings were made of the rupture patterns, as shown in Fig. 12. Staining protocols used for each lens are listed in Table 1.

When the incision was made with the blade perpendicular to the equator, the borders of the incision typically remained closed (e.g. lenses P1, P2, P3, P4 in Fig. 12); this indicates an absence of significant tensile circumferential stresses (consistent with the inflated capsule model). Conversely, when the blade was parallel to the equator (lenses P5, P6, P7, P8, P9) in some (but not all) cases the edges of the capsule tended to open. Consistent with the inflated capsule model, this suggests the existence of meridional residual stresses in the capsule. In some cases when a parallel cut was made, the capsule suffered a vertical rupture that ran to the anterior and the posterior surfaces. 
Although the puncture test images presented here exhibit behavior that is broadly consistent with the inflated membrane model, the results are not regarded as being conclusive. It seems likely that, for presbyopic lenses (in contrast to young lenses), the lens substance is sufficiently stiff for the inflated membrane model (which relies on the lens substance being soft relative to the capsule) to be unrealistic. For presbyopic lenses, therefore, the inflated membrane model may not provide an appropriate representation of the residual stresses in the capsule.

\section{Conclusions}

Evidence has been assembled to demonstrate the presence of equatorial wrinkles in the isolated human lens. A mechanical model of the young lens (in which the substance is relatively soft) is proposed in which the presence of equatorial wrinkles is recognised as being a consequence of the action of an excess intralenticular pressure. The existence of equatorial wrinkles has been noted in previous publications, but, to the authors' knowledge, this is the first study that has sought to investigate the geometric arrangement of the wrinkles and to suggest a mechanism that may be responsible for their formation.

It is not possible, on the basis of the evidence presented in the current paper, to provide a complete description of equatorial wrinkling. However, it is demonstrated in the paper via measurements of wrinkles on the surface of post mortem lenses, that equatorial wrinkles are consistent with a simple mechanical model of the lens consisting of a stiff membrane (the capsule) surrounding a fluid (the lens substance). A model of this sort is only likely to be appropriate for the young lens (for which the lens substance is relatively soft). It seems plausible that equatorial wrinkles initially develop within the young lens, on the basis of the mechanism suggested in the current paper. As the lens ages and the lens substance stiffens, the lens substance becomes moulded to the wrinkled shape of the capsule. The current results, for example, indicate that when a presbyopic lens is de-capsulated, the lens substance retains the appearance of equatorial wrinkles. When a presbyopic lens is subjected to a puncture test, the results show some evidence of meridional residual stresses (and the absence of circumferential residual stresses) but the results are inconclusive. This is perhaps unsurprising as the residual stresses in the capsule of a presbyopic lens are unlikely to correspond directly to those in a young lens, for which the inflated capsule model is thought to provide a reasonable representation of behaviour.

The presence of equatorial wrinkles may have an influence on the mechanics of the accommodation process. It may therefore be necessary for detailed consideration to be given to the mechanical significance of equatorial wrinkles in any future, improved, mechanical models of accommodation and presbyopia. 


\section{Appendix - Geometric analysis of the lens images.}

The procedure that has been adopted to estimate the elevation angles (in lens polar coordinates) corresponding to the wrinkles observed in the images collected using the lens rotation box is described below. This procedure is based on the assumption that the lens is a solid of revolution with the sagittal profile defined by the outlines given in Urs et al. (2010). Coordinate systems used in the analysis are shown in Figs. 7 and 8.

The surface of the lens (from Urs et al. 2010), for the case where the anterior pole is placed uppermost, is given by,

$$
f(\rho, \theta)=\rho-\sum_{n=0}^{10}\left(A_{n 1}+A_{n 2} \times a g e\right) \cos (n(\pi-\theta))=0
$$

where $(\rho, \theta)$ are spherical polar coordinates defined in the lens coordinate system, where $\theta=0$ corresponds to the anterior pole and $\theta=\pi$ corresponds to the posterior pole.

At a particular value of lens rotation $\psi_{r}$, a wrinkle is identified at a point on the lens outline at azimuthal angle $\phi^{\prime \prime}$ (in the shaft coordinate system, as viewed by the camera). It is desired to compute the elevation angle $\beta$ in lens coordinates that corresponds to this point. Rotation of coordinates gives $X=R_{\text {tilt }} X^{\prime}$ where $R_{\text {tilt }}$ is a rotation matrix that rotates $X$ by the tilt angle, $\alpha$, so that coordinate direction $x^{\prime}$ is aligned with the shaft axis of rotation. $R_{\text {tilt }}$ is,

$$
R_{t i l t}=\left[\begin{array}{ccc}
\cos \alpha & 0 & \sin \alpha \\
0 & 1 & 0 \\
-\sin \alpha & 0 & \cos \alpha
\end{array}\right]
$$

The value of the tilt angle is determined from the sagittal lens image for $\psi_{r}=-90^{\circ}$. A positive tilt angle corresponds to a clockwise rotation of the lens with respect to $X^{\prime \prime}$ coordinate system, as indicated in Fig 7 . The lens rotation shaft is rotated by angle $\psi_{r}$. This corresponds to a coordinate transformation $X^{\prime}=R_{\text {rot }} X^{\prime \prime}$, where,

$$
R_{\text {rot }}=\left[\begin{array}{ccc}
1 & 0 & 0 \\
0 & \cos \psi_{r} & \sin \psi_{r} \\
0 & -\sin \psi_{r} & \cos \psi_{r}
\end{array}\right]
$$

A wrinkle is observed on the lens edge at Point W with azimuthal angle $\phi^{\prime \prime}$ when the lens rotation angle is $\psi_{r}$. To determine the polar angle in lens coordinates, corresponding to Point $\mathrm{W}$, the following plane (on which Point $\mathrm{W}$ lies) is considered,

$$
x^{\prime \prime}=\lambda \cos \phi^{\prime \prime}, y^{\prime \prime}=\lambda \sin \phi^{\prime \prime}, \quad z^{\prime \prime}=\mu
$$

where $\lambda$ and $\mu$ are scalar variables. Values of $(\lambda, \mu)$ corresponding to Point $W$ are determined by maximising $\lambda$ subject to: 


$$
\begin{gathered}
X=R_{\text {tilt }} R_{\text {rot }} X^{\prime \prime} \\
\rho^{2}=x^{2}+y^{2}+z^{2} \\
\tan \theta=\frac{\sqrt{x^{2}+y^{2}}}{z} \\
f(\rho, \theta) \leq 0
\end{gathered}
$$

Once $(\lambda, \mu)$ have been determined, appropriate coordinate transformations are used to find coordinates of Point $\mathrm{W},(\beta, \phi)$, in the lens coordinate system.

\section{References}

Augusteyn, R.C., 2008. Growth of the lens: In vitro observations. Clinical and Experimental Optometry 91, 226-239.

Bron, A.J., Tripathi, R.C., Tripathi, B.J., 1997. Wolff's Anatomy of the Eye and Orbit, 8th ed.

Burd, H.J., Judge, S.J., Cross, J.A., 2002. Numerical modelling of the accommodating lens. Vision Research 42 (18), 2235-2251.

Burd, H.J., Regueiro, R.A., 2015. Finite element implementation of a multiscale model of the human lens capsule. Biomechanics and Modeling in Mechanobiology, 1-16.

Burd, H.J., Wilde, G.S., Judge, S.J., 2006. Can reliable values of Young's modulus be deduced from Fisher's (1971) spinning lens measurements? Vision Research 46, 1346-1360.

Burd, H.J., Wilde, G.S., Judge, S.J., 2011. An improved spinning lens test to determine the stiffness of the human lens. Experimental Eye Research 92, 28-39.

Chylack, L.T., Wolfe, J.K., Singer, D.M., Leske, M.C., Bullimore, M.A., Bailey, I.L., Friend, J., McCarthy, D., Wu, S.-Y., 1993. The lens opacities classification system III. Archives of ophthalmology 111, 831-836.

Fisher, R.F., 1969. Elastic constants of the human lens capsule. Journal of Physiology 201, 1-19.

Fisher, R.F., 1971. The elastic constants of the human lens. Journal of Physiology 212, 147-180.

Hermans, E.A., Dubbelman, M., van der Heijde, G.L., Heethaar, R.M., 2006. Estimating the external force acting on the human eye lens during accommodation by finite element modelling. Vision Research 46, 3642-3650.

Hermans, E.A., Dubbelman, M., van der Heijde, G.L., Heethaar, R.M., 2008. Change in the accommodative force on the lens of the human eye with age. Vision Research 48, 119-126.

Irvine, H.M., 1981. Cable structures. MIT Press

Krag, S., Andreassen, T.T., 1996. Biomechanical measurements of the porcine lens capsule. Experimental Eye Research 62, 253-260.

Krag, S., Andreassen, T.T., 2003. Mechanical properties of the human lens capsule. Progress in Retinal and Eye Research 22, 749-767.

McCulloch, C., 1954. The zonule of zinn: its origin, course, and insertion, and its relation to neighbouring structures. Trans Am Ophthalmol Soc. 52, 525-585.

Michael, R., Barraquer, R.I., Willekens, B., van Marle, J., Vrensen, G.F.J.M., 2008. Morphology of agerelated cuneiform cortical cataracts: The case for mechanical stress. Vision Research 48, 626-634. 
Pedrigi, R.M., David, G., Dziezyc, J., Humphrey, J.D., 2007. Regional mechanical properties and stress analysis of the human anterior lens capsule. Vision Research 47, 1781-1789.

Rosen, A.M., Denham, D.B., Fernandez, V., Borja, D., Ho, A., Manns, F., Parel, J.-M., Augusteyn, R.C., 2006. In vitro dimensions and curvatures of human lenses. Vision Research 46, 1002-1009.

Stachs, O., Martin, H., Behrend, D., Schmitz, K.P., Guthoff, R., 2006. Three-dimensional ultrasound biomicroscopy, environmental and conventional scanning electron microscopy investigations of the human zonula ciliaris for numerical modelling of accommodation. Graefe's Archive for Clinical and Experimental Ophthalmology 244, 836-844.

Urs, R., Manns, F., Ho, A., Borja, D., Amelinckx, A., Smith, J., Jain, R., Augusteyn, R., Parel, J.M., 2009. Shape of the isolated ex-vivo human crystalline lens. Vision Research 49, 74-83.

Urs, R., Ho, A., Manns, F., Parel, J.-M., 2010. Age-dependent Fourier model of the shape of the isolated ex vivo human crystalline lens. Vision Research 50, 1041-1047.

Weeber, H.A., van der Heijde, G.L., 2007. On the relationship between lens stiffness and accommodative amplitude. Experimental Eye Research 85, 602-607.

Wilde, G.S., Burd, H.J., Judge, S.J., 2012. Shear modulus data for the human lens determined from a spinning lens test. Experimental Eye Research 97, 36-48. 


\section{Table 1}

Specification of the lenses tested in the lens rotation box (L1-L4) and in the puncture test (P1-P9). Wrinkle grade refers to a visual assessment of the number of wrinkles observed around the equator of the lens (see Fig. 4). Red ink (Edding T 100, Edding International GmbH) and methylene blue were employed as stains as indicated.

\begin{tabular}{ccccc}
\hline Lens reference & Age (years) & $\begin{array}{c}\text { Time post } \\
\text { mortem (days) }\end{array}$ & Wrinkle grade & Staining \\
\hline L1 & 61 & 6 & 2 & - \\
\hline L2 & 66 & 4 & 3 & - \\
\hline L3 & 82 & 4 & 3 & - \\
\hline L4 & 78 & 6 & 3 & methylene blue \\
\hline P1 & 66 & 4 & 1 & methylene blue \\
\hline P2 & 77 & 3 & 2 & red ink \\
\hline P3 & 90 & 5 & 1 & red ink \\
\hline P4 & 77 & 5 & 2 & red ink \\
\hline P5 & 85 & 6 & 3 & red ink \\
\hline P6 & 88 & 1 & 1 & methylene blue \\
\hline P7 & 46 & 5 & 1 & red ink \\
\hline P8 & 84 & 6 & 3 & methylene blue \\
\hline P9 & 90 & 3 & 1 & \\
\hline
\end{tabular}

Table 2

Data from the lens rotation box study: number of wrinkle peaks detected in the coronal image, $\psi_{r}=$ 0 for $\phi=90^{\circ} \pm 50^{\circ}$, wavelength and wrinkle angle.

\begin{tabular}{|c|c|c|c|c|}
\hline \multirow[t]{2}{*}{ Lens Reference } & \multirow[t]{2}{*}{ No. of wrinkles } & \multirow{2}{*}{$\begin{array}{c}\text { Minimum and } \\
\text { maximum } \\
\text { wavelength }\end{array}$} & \multicolumn{2}{|c|}{ Wrinkle angles } \\
\hline & & & Anterior & Posterior \\
\hline L1 & 12 & $6^{\circ} ; 9^{\circ}$ & $12^{\circ}$ & $8^{\circ}$ \\
\hline $\mathrm{L} 2$ & 8 & $6^{0} ; 14^{0}$ & $5^{0}$ & $4^{0}$ \\
\hline L3 & 8 & $7^{0} ; 12^{0}$ & $6^{0}$ & $4^{0}$ \\
\hline L4 & 4 & $6^{0} ; 7^{0}$ & $7^{0}$ & $3^{\circ}$ \\
\hline
\end{tabular}




\section{Figure Captions}

Fig. 1. Images of an isolated 63-year lens from (reprinted from Rosen et al., 2006 with permission from Elsevier). Note that the sagittal outline (left) is smooth and the coronal outline (right) is undulating. This is consistent with the proposed concept of equatorial wrinkling.

Fig. 2. Stress resultants acting in the lens capsule.

Fig. 3. Capsule wrinkling model. In zone I the capsule is taut. In zone II the capsule is wrinkled.

Fig. 4. Grading of coronal images of isolated lenses according to wrinkle quantity. Of the 185 lenses that were inspected, 64 lenses had less than 5 wrinkles, 58 lenses had between 5 and 10 wrinkles, 32 lenses had between 10 and 15 wrinkles, and 31 lenses had more than 15 wrinkles. None of the lenses were observed to be wrinkle-free.

Fig. 5. Observations on wrinkle grade grouped according to age (left), cortical cataract (centre) and nuclear cataract (right); $n$ is the number of lenses within each group.

Fig. 6. Side view of the lens rotation box.

Fig. 7. Fig. (a) shows the 'box coordinate system' $X^{\prime \prime \prime}=\left(x^{\prime \prime \prime}, y^{\prime \prime \prime}, z^{\prime \prime \prime}\right)$ which is fixed to the lens box. Coordinate direction $x^{\prime \prime \prime}$ is aligned with the shaft axis of rotation; direction $z^{\prime \prime \prime}$ is vertically upwards. The 'shaft coordinate system' $X^{\prime \prime}=\left(x^{\prime \prime}, y^{\prime \prime}, z^{\prime \prime}\right)$, shown in (b), is aligned with the box coordinate system, but translated so that the origin is at point $C$ (defined as the intersection of the polar axis and the equatorial plane of the lens). The 'lens-shaft coordinate system' $X^{\prime}=\left(x^{\prime}, y^{\prime}, z^{\prime}\right)$ is fixed to the lens and aligned such that for lens rotation angle $\psi_{r}=0$ the $X^{\prime}$ and $X^{\prime \prime}$ axes coincide. The 'lens coordinate system' $X=(x, y, z)$ is fixed to the lens, with origin at $C$ and aligned with the principal axes of the lens. The axes $X^{\prime}$ and $X$ are related by a rotation $\alpha$ (where $\alpha$ is the tilt angle) about the $y^{\prime}$ axis.

Fig. 8. Convention adopted for spherical coordinates representing an arbitrary point $\mathrm{P}$, where $\theta$ is the polar angle, $\phi$ is the azimuthal angle and $\beta=\pi / 2-\theta$ is the elevation angle.

Fig. 9. Dark field images of lens L2 indicating the shaft coordinate system. In images (a), (b) and (c) the lens is attached to the cradle at the posterior pole; (a) $\psi_{r}=0$ (b) $\psi_{r}=-46.8^{\circ}$ and (c) $\psi_{r}=$ $\left.-90^{\circ}\right)$.

Fig. 10. Data for lens L2. (a) Filtered lens edge profiles for the anterior surface at $3.6^{\circ}$ increments of lens rotation angle, $\psi_{r}$. Wrinkles (prominence $=1.75$ ) are shown with filled circles. (b) Wrinkle locations plotted in the lens coordinate system. The solid lines represent the locus of the lens outline as viewed by the camera, at fixed values of lens rotation angle, $\psi_{r}$; these lines are spaced at a constant angular increment of $3.6^{\circ}$.

Fig. 11. Equatorial wrinkles seen in lens $L 3$ Intact lens (left) and de-capsulated lens (right).

Fig. 12. The V-shape symmetrical lancet used in the puncture test (left); schematic of the orientations of the incisions made to the capsule (middle) and schematic drawing of rupture patterns after incision of the capsule (right). Incisions perpendicular to the equator (above) and parallel to the equator (below). 


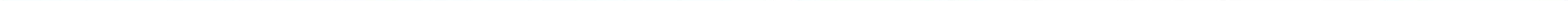




\section{Small capsule}

element

\section{Polar axis}

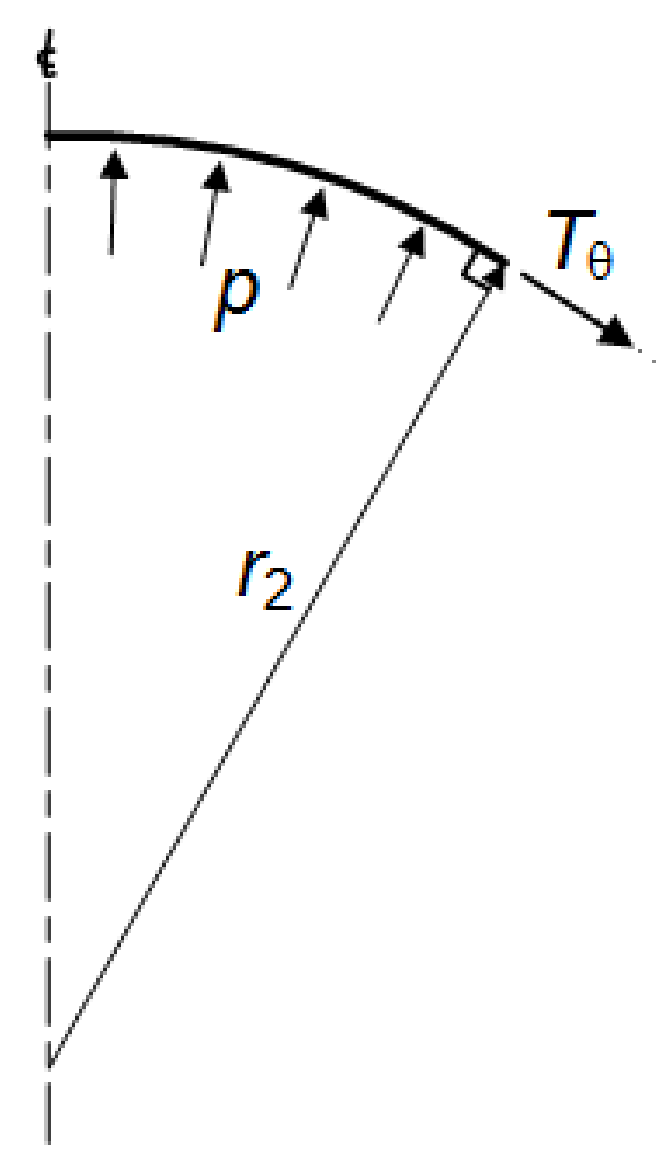

(a)

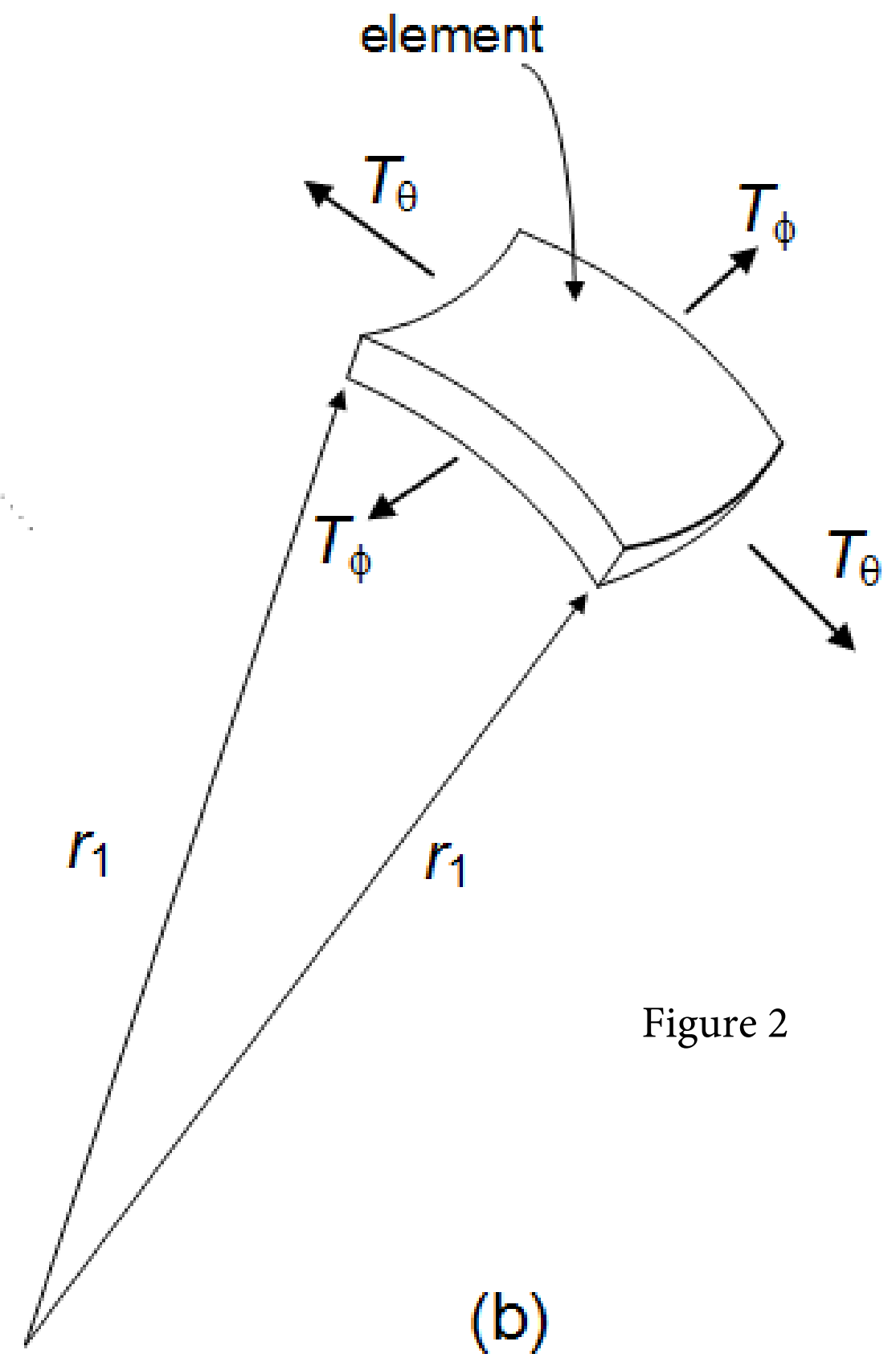




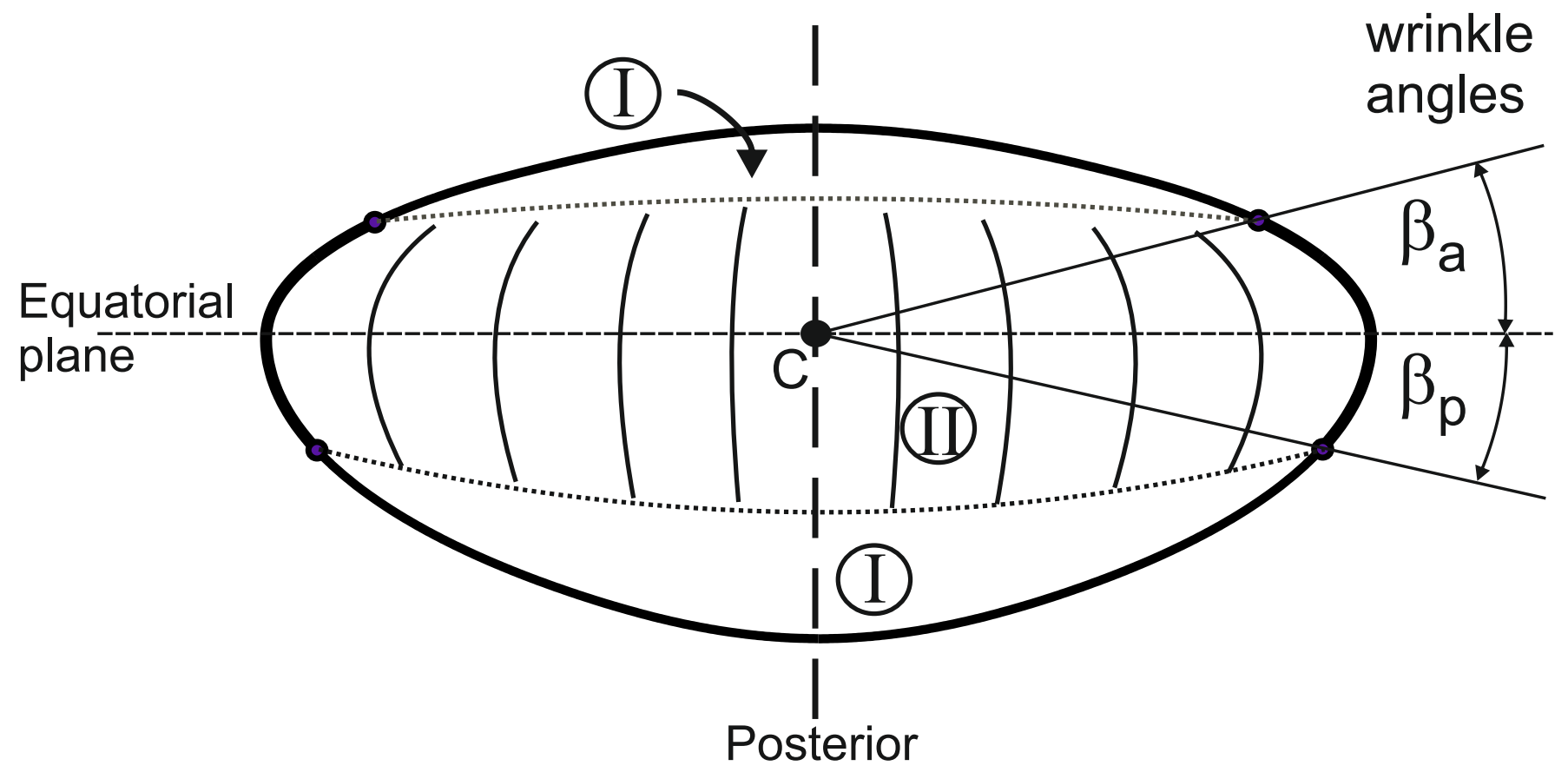

Figure 3 
Grade 1 Figure 4 $<5$ wrinkles
Grade 2

5-10 wrinkles
Grade 3 $10-15$ wrinkles
Grade 4 $>15$ wrinkles 

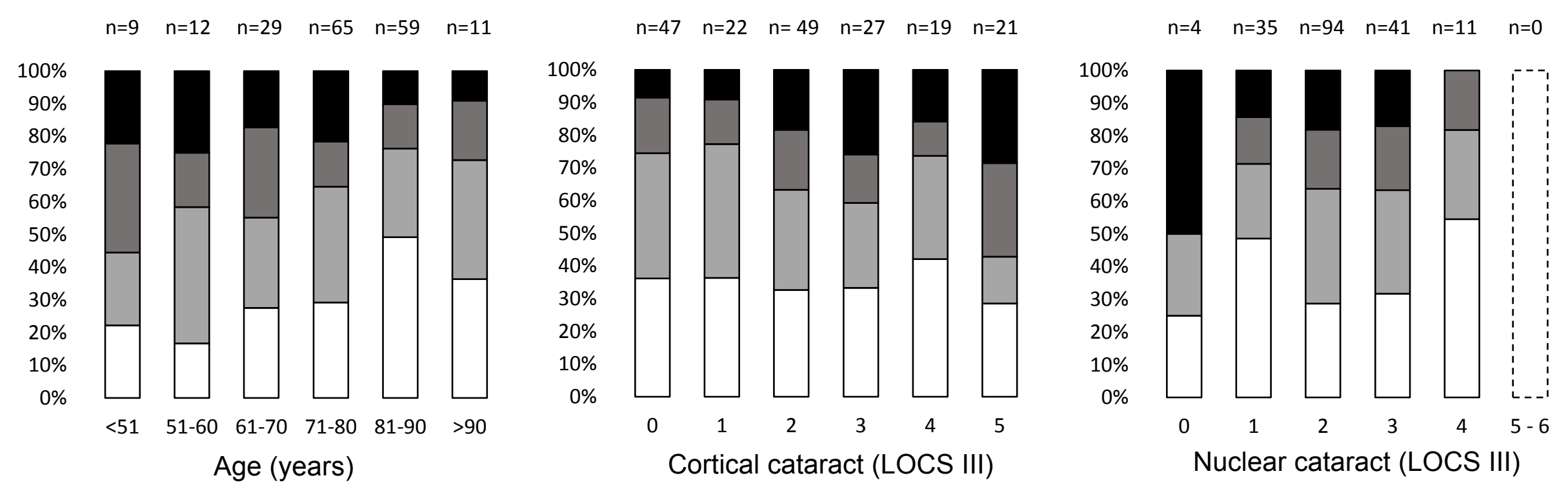

Wrinkle grade

Grade 4

$\square$ Grade 3

$\square$ Grade 2

$\square$ Grade 1

Figure 5 


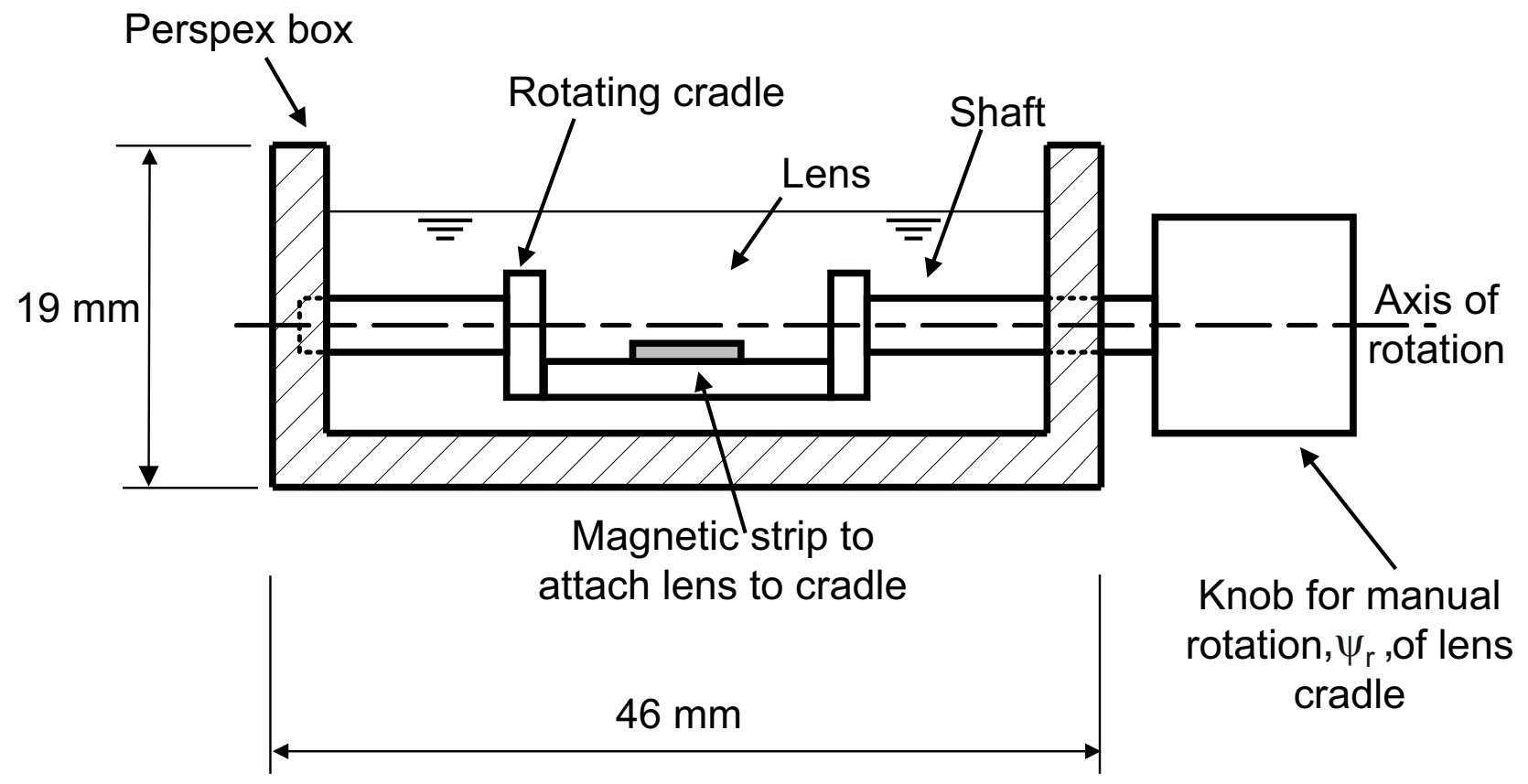

Figure 6 
(a)

(b)

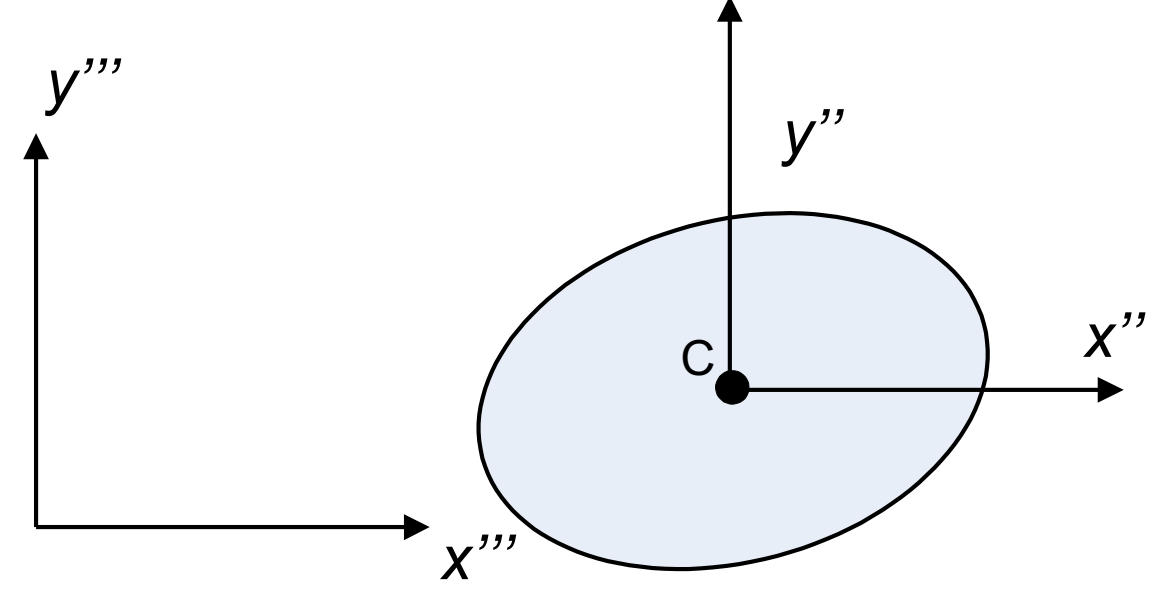

(c)

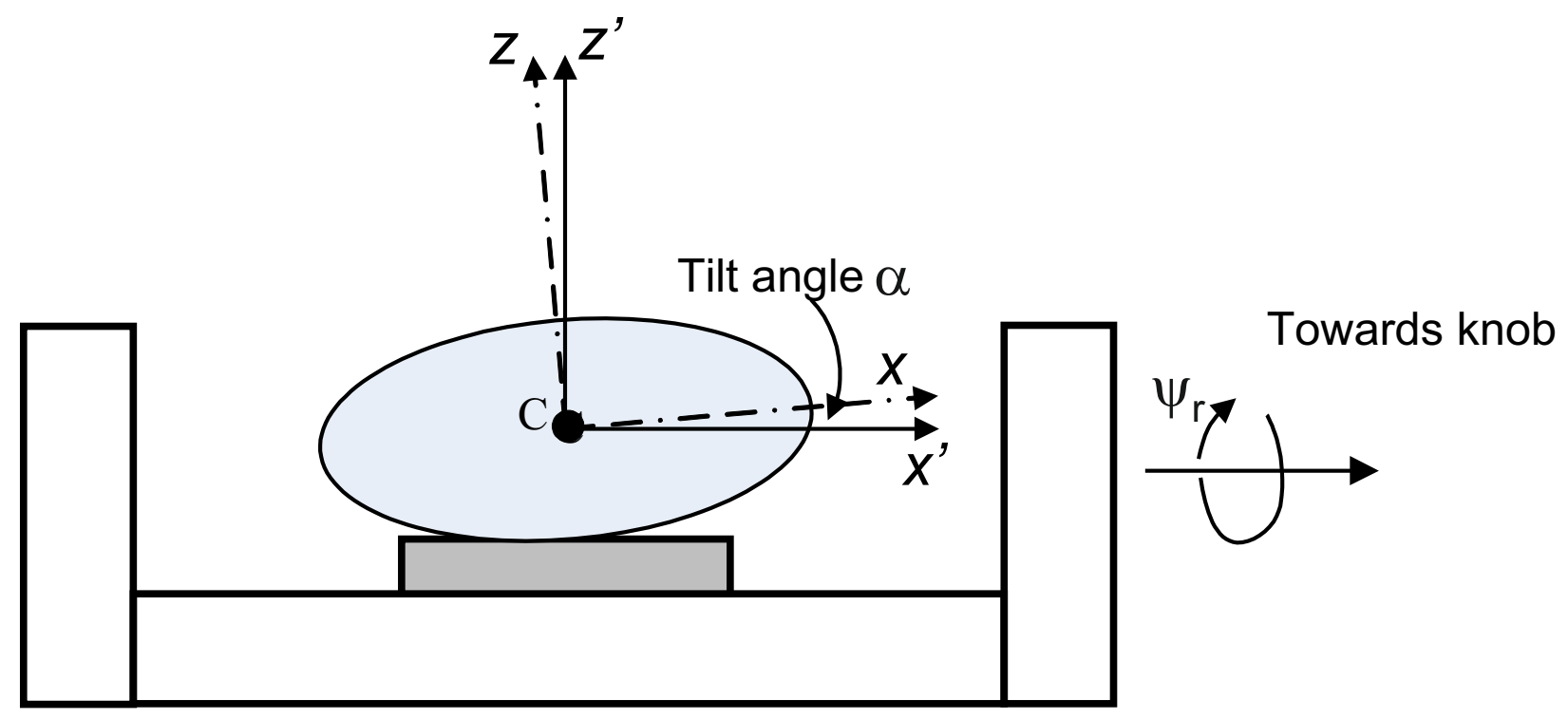

Figure 7 


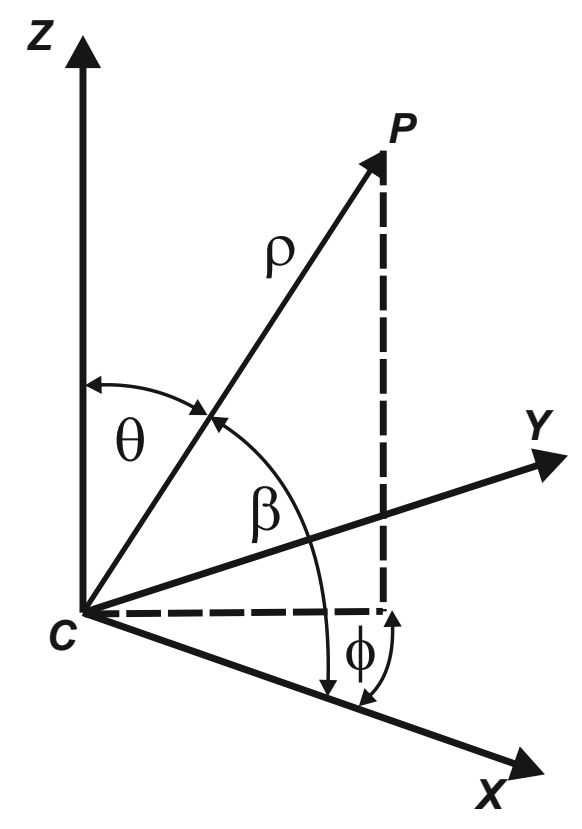

Figure 8 
(a)

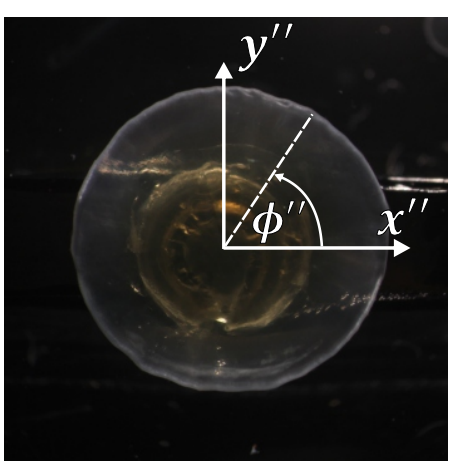

(b)

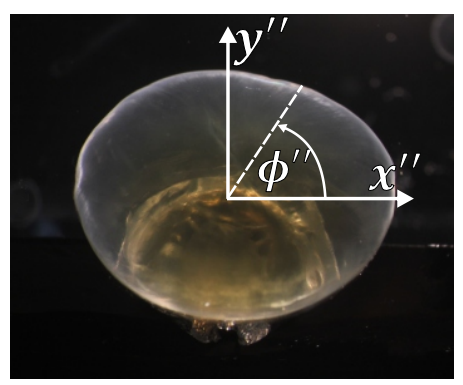

(c)

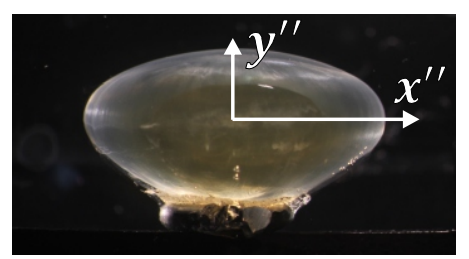

Figure 9 


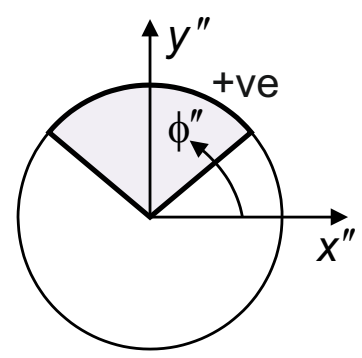

Azimuthal angle

(a)

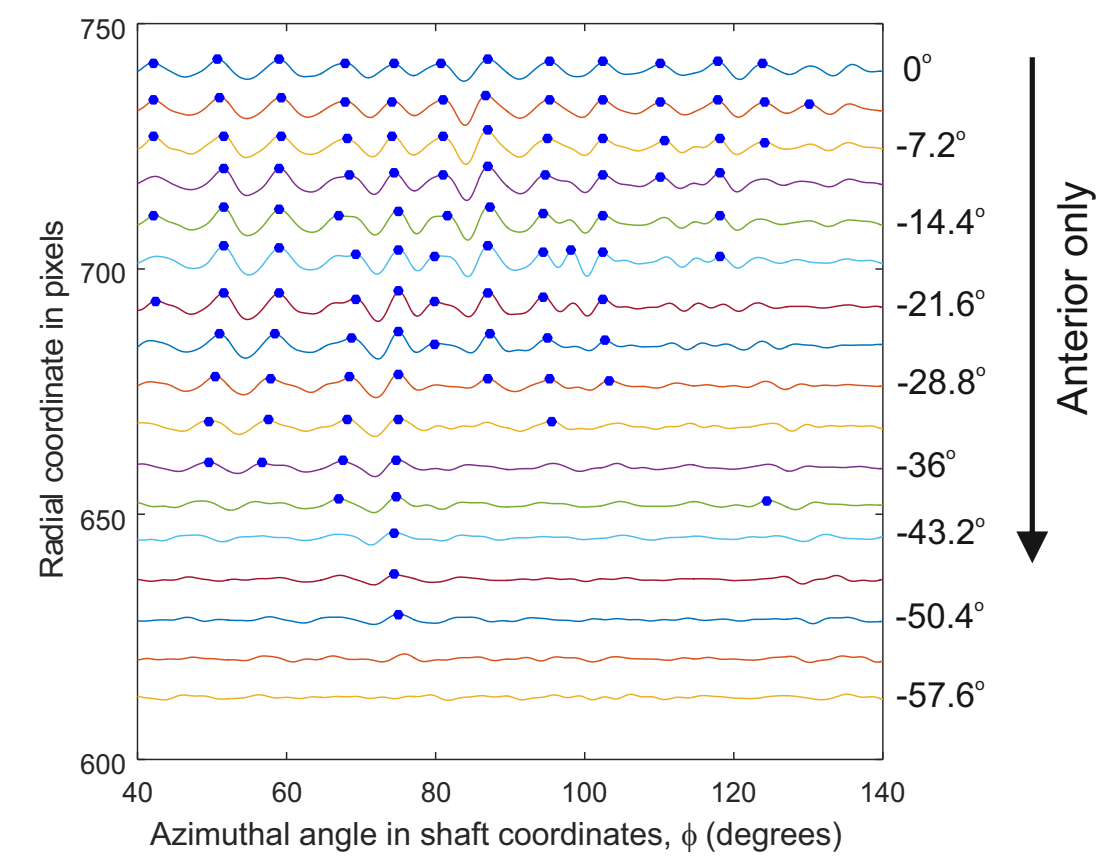

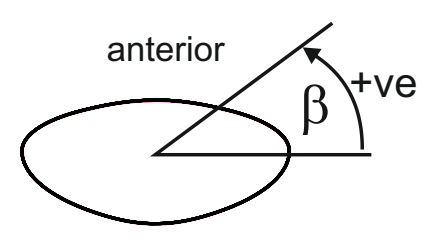

posterior

Elevation angle

(b)

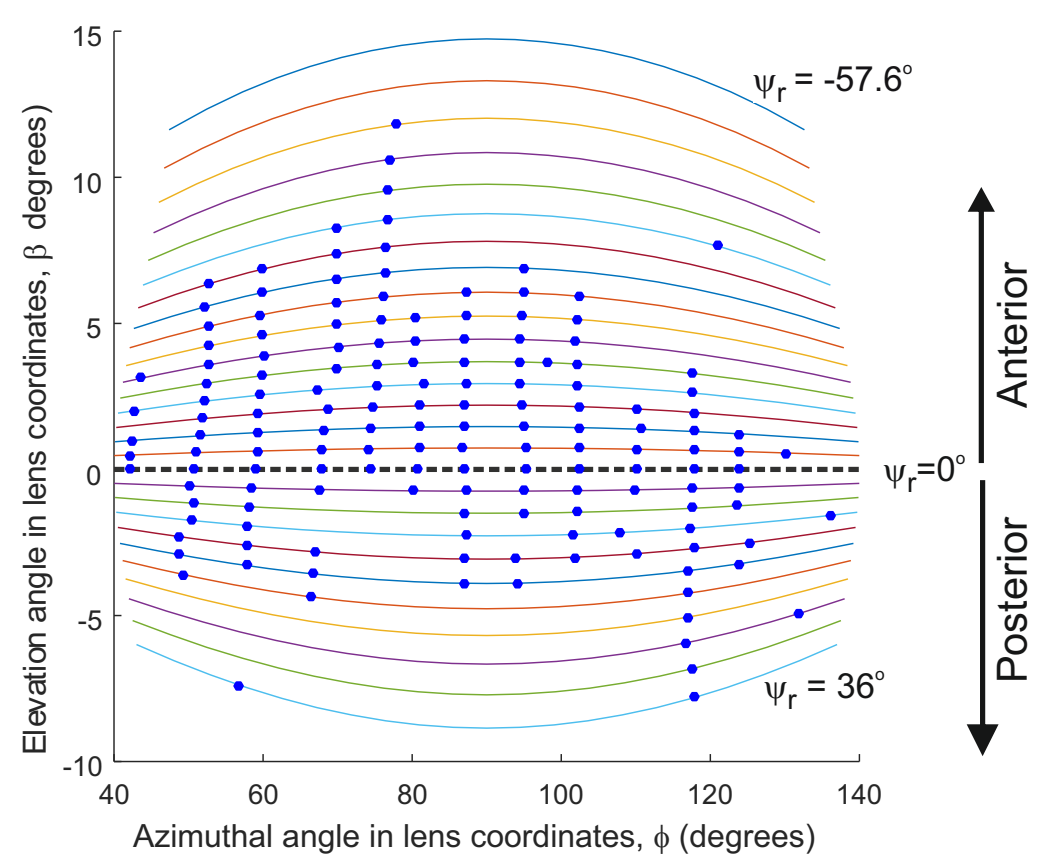

Figure 10 

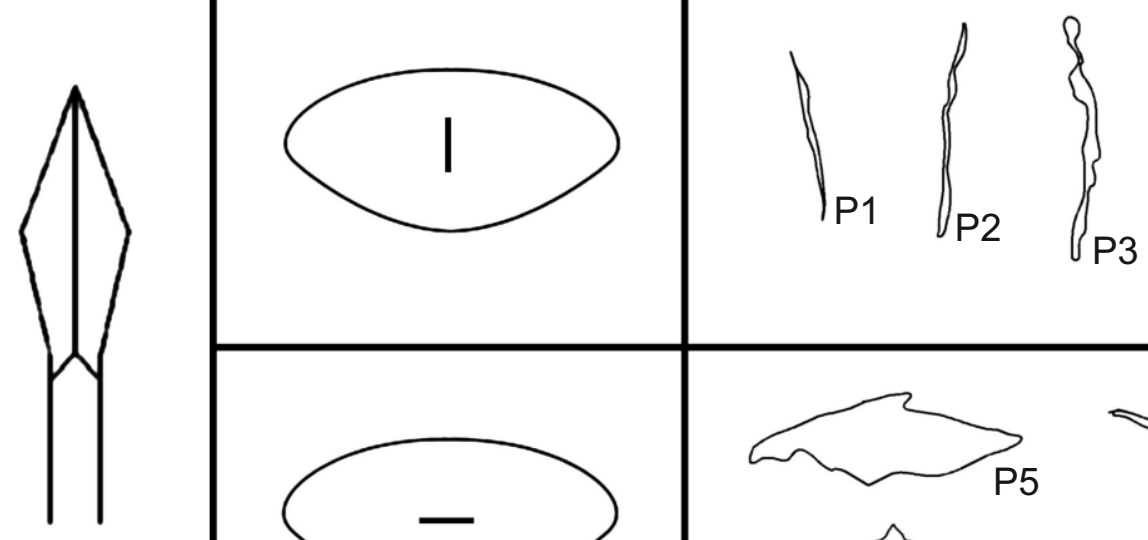

Figure 12
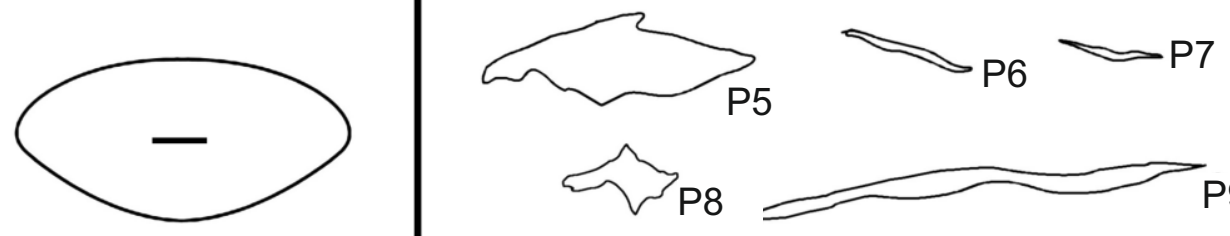

$500 \mu \mathrm{m}$ 